\title{
Pancreatic neuro-insular network in young mice revealed by $3 D$ panoramic histology
}

\author{
Shiue-Cheng Tang ${ }^{1,2,3}$ - Chia-Ning Shen ${ }^{4,5}$ - Pei-Yu Lin ${ }^{4,5}$ - Shih-Jung Peng ${ }^{1,2}$ • \\ Hung-Jen Chien ${ }^{2} \cdot$ Ya-Hsien Chou ${ }^{2}$. Chester E. Chamberlain ${ }^{6}$ • Pankaj J. Pasricha ${ }^{7}$
}

Received: 23 February 2017 / Accepted: 4 July 2017 / Published online: 1 September 2017

(C) Springer-Verlag GmbH Germany 2017

\begin{abstract}
Aims/hypothesis It has been proposed that the neuro-insular network enables rapid, synchronised insulin secretion. However, to date, acquiring the pancreatic tissue map to study the neural network remains a challenging task as there is a lack of feasible approaches for large-scale tissue analysis at the organ level. Here, we have developed 3-dimensional (3D) panoramic histology to characterise the pancreatic neuroinsular network in young mice.

Methods Pancreases harvested from young wild-type B6 mice ( 3 and 8 weeks old) and $d b / d b$ mice ( 3 weeks old; $d b / d b$ vs $d b /+$ ) were used to develop 3D panoramic histology. Transparent pancreases were prepared by optical clearing to enable deeptissue, tile-scanning microscopy for qualitative and quantitative analyses of islets and the pancreatic tissue network in space. Results 3D panoramic histology reveals the pancreatic neurovascular network and the coupling of ganglionic and islet populations via the network. This integration is identified in both 3-and 8-week-old mice, featuring the peri-arteriolar
\end{abstract}

neuro-insular network and islet-ganglionic aggregation. In weaning hyperphagic $d b / d b$ mice, the $3 \mathrm{D}$ image data identifies the associated increases in weight, adipose tissue attached to the pancreas, density of large islets (major axis $>150 \mu \mathrm{m}$ ) and pancreatic sympathetic innervation compared with $d b /+$ mice. Conclusions/interpretation Our work provides insight into the neuro-insular integration at the organ level and demonstrates a new approach for investigating previously unknown details of the pancreatic tissue network in health and disease.

Keywords 3D histology · Ganglion · Insulin · Islet · Neural network · Obesity $\cdot$ Sympathetic nerve

$\begin{array}{ll}\text { Abbreviations } \\ \text { 3D } & \text { 3-Dimensional } \\ \text { TH } & \text { Tyrosine hydroxylase } \\ \text { vAChT } & \text { Vesicular acetylcholine transporter }\end{array}$

Pei-Yu Lin and Shih-Jung Peng contributed equally to this work.

Electronic supplementary material The online version of this article (doi:10.1007/s00125-017-4408-y) contains peer-reviewed but unedited supplementary material, which is available to authorised users.

Shiue-Cheng Tang

sctang@life.nthu.edu.tw

$\triangle$ Chia-Ning Shen

cnshen@gate.sinica.edu.tw

1 Connectomics Research Center, National Tsing Hua University, Hsinchu, Taiwan

2 Institute of Biotechnology, National Tsing Hua University, Hsinchu, Taiwan
3 Department of Medical Science, National Tsing Hua University, 101, Sec. 2, Kuang Fu Rd, Hsinchu 30013, Taiwan

4 Genomics Research Center, Academia Sinica, 128, Sec. 2, Academia Rd, Taipei 11529, Taiwan

5 Graduate Institute of Life Sciences, National Defense Medical Center, Taipei, Taiwan

6 Diabetes Center, University of California at San Francisco, San Francisco, CA, USA

7 Johns Hopkins Center for Neurogastroenterology, Johns Hopkins University School of Medicine, Baltimore, MD, USA 


\section{Introduction}

Pancreatic microstructures were first detailed by Paul Langerhans in his thesis 'Contributions to the microscopic anatomy of the pancreas' [1]. However, to date, investigators have continued to be limited by standard microtome-based histology, where the pancreatic specimen is sliced into thin sections $(3-5 \mu \mathrm{m})$ to characterise the tissue morphology. Due to this technical limitation, the pancreatic neurovascular networks and their association with the endocrine islets can only be examined locally without substantial understanding of the network structure at the organ level.

Scattered in the pancreas, the endocrine islets rely on neurovascular inputs for modulation of hormone secretion including fasting glucagon, and cephalic and postprandial insulin secretion $[2,3]$. Although the secretory cues from the circulatory pathway (e.g. nutrients and circulating factors) have been well characterised [4], details of how the pancreatic neural network connects to and governs the scattered islets (approximately one million in an adult human pancreas and thousands in a rodent pancreas) are still not completely understood. Despite the lack of information, evidence has emerged that indicates the functional importance and the intrinsic association of islets with the neural network in the pancreas.

First, at the functional level, pulsatile insulin secretion from the entire pancreas (during a period of 5-15 $\mathrm{min}$ ) requires secretion from individual islets to be synchronised as a unit [5-7]. The leading hypothesis explaining this coordinated insulin release suggests that the intra-pancreatic neural network connects the islets and synchronises secretion $[8,9]$. Importantly, it is currently unknown whether the loss of insulin oscillations in type 2 diabetes is associated with the neural network dysfunction [9-11]. Second, in humans, cephalic insulin release depends on the neural pathway and the response is important for postprandial glucose tolerance [3]. Third, in mouse development, the pancreatic/islet sympathetic innervation is important for islet formation and functional maturation [12]. Fourth, in mouse islet transplantation, the engrafted islets are able to remodel the surrounding sympathetic nerves and the associated glial cells to regain the islet innervation patterns $[13,14]$, suggesting an intrinsic ability of islets to recruit and associate with neural tissues.

On the basis of the established functional results and the intrinsic neural-islet association, study of the islet microenvironment should ideally include the neural tissues to integrate the local islet microenvironment with the surrounding pancreatic neural network. From a therapeutic perspective, reaching islets through the neural pathways represents a natural route to target islets for potential diabetes treatment. However, tissue maps of the pancreatic neural network and its association with the islets are missing. This is largely due to the dispersed nature of the neural network and the scattered islets in the pancreas, which cannot easily be portrayed by standard histology in order to characterise their patterns and relationship in space.

To map the pancreatic and islet innervation in this research we prepared transparent pancreases $[15,16]$ with labelled islets, nerves and blood vessels for 3-dimensional (3D) panoramic histology. Qualitative and quantitative analyses of the islet and ganglionic populations and their associations, the neuro-insular network and neural network remodelling in disease are presented and discussed here.

\section{Methods}

Animals Normal pancreases harvested from 3-week-old (weaning) and 8-week-old wild-type C57BL/6 (B6) mice (National Laboratory Animal Center, Taipei, Taiwan) were used to develop 3D pancreatic histology. Diseased pancreases harvested from 3-week-old $d b / d b$ (control: $d b /+$ heterozygous) mice were used to examine pancreatic neural network remodelling. Animals were kept in a temperature-controlled and pathogen-free facility with a 12:12 h light:dark cycle and ad libitum access to food and water. The Institutional Animal Care and Use Committees at National Tsing Hua University and Academia Sinica approved all procedures with mice. Overall, seven 3-week-old B6, five 8-week-old B6, five $d b$ / $d b$ and four $d b /+$ mice were randomly selected from their age and $d b$ populations and used to generate the representative images, including two complete sets of whole pancreas images from two 3-week-old B6 mice.

Tissue labelling and microscopy Pancreatic blood vessels were labelled with cardiac perfusion of lectin-Alexa Fluor 488 conjugates (Invitrogen, Carlsbad, CA, USA) followed by $4 \%$ paraformaldehyde perfusion fixation [17]. The pancreas was then harvested and post-fixed in $4 \%$ paraformaldehyde solution for $40 \mathrm{~min}$ at $15^{\circ} \mathrm{C}$. Vibratome sections of the fixed tissue $(\sim 500 \mu \mathrm{m})$ were then immersed in $2 \%$ Triton X-100 solution for $2 \mathrm{~h}$ at $15^{\circ} \mathrm{C}$ for permeabilisation.

Five different primary antibodies were used to immunolabel the tissues, as per manufacturer's instructions. The antibodies used were guinea pig anti-insulin (GTX27842, GeneTex, Irvine, CA, USA), rabbit anti-TUJ1 (neuronal marker; PRB-435P, Covance, Princeton, NJ, USA), rabbit anti-PGP9.5 (neuronal marker; 2932-1, Epitomics, Burlingame, CA, USA), rabbit anti-vesicular acetylcholine transporter (vAChT, parasympathetic marker; 139103, Synaptic Systems, Goettingen, Germany) and rabbit anti-tyrosine hydroxylase (TH, sympathetic marker; AB152; Millipore, Billerica, MA, USA) antibodies. Before applying the antibody, the tissue was rinsed in PBS. This was followed by a blocking step, incubating the tissue with the blocking buffer ( $2 \%$ Triton X-100, $10 \%$ normal goat serum and $0.02 \%$ sodium azide in PBS). The primary antibody was then diluted in the 
dilution buffer $(1: 100,0.25 \%$ Triton X-100, $1 \%$ normal goat serum and $0.02 \%$ sodium azide in $\mathrm{PBS}$ ) to replace the blocking buffer and incubated for 1 day at $15^{\circ} \mathrm{C}$.

Alexa Fluor 647 conjugated goat anti-rabbit secondary antibody and Alexa Fluor 546 conjugated goat anti-guinea pig secondary antibody (1:200, Invitrogen) were used to reveal the immunostained structures. Propidium iodide staining (50 $\mathrm{\mu g} / \mathrm{ml}$, Invitrogen) was performed to reveal the nuclei. The labelled specimens were immersed in the optical clearing aqueous solution (RapiClear 1.52 solution [18, 19], SunJin Lab, Hsinchu, Taiwan) 1 day before being imaged via confocal microscopy.

Imaging of the tissue structure was performed with a Zeiss LSM 510 Meta confocal microscope (Carl Zeiss, Jena, Germany) equipped with $\times 10$ and $\times 20$ Fluar, $\times 25$ LD PlanApochromat and $\times 40$ LD C-Apochromat lenses under the tilescan mode with automatic image stitching. The laser-scanning process was operated under the multi-track scanning mode to acquire signals, including transmitted light signals. The Alexa Fluor 647-labelled structures were excited at $633 \mathrm{~nm}$ and the fluorescence was collected by the $650-710 \mathrm{~nm}$ band-pass filter. The propidium iodide-labelled nuclei and Alexa Fluor 546-labelled structures were excited at $543 \mathrm{~nm}$ and the signals were collected by the $560-615 \mathrm{~nm}$ band-pass filter. The lectinAlexa Fluor 488-labelled blood vessels were excited at $488 \mathrm{~nm}$ and the fluorescence was collected by the 500 $550 \mathrm{~nm}$ band-pass filter.

Image projection and analysis The Avizo 6.2 image reconstruction software (VSG, Burlington, MA, USA), Zen software (Carl Zeiss) and LSM 510 software (Carl Zeiss) were used for projection, signal segmentation, noise reduction (Gaussian filter; kernel $3 \times 3$ ) and analysis of confocal images. Percentage of adipose tissue in the pancreatic section was estimated by the black areas (pixels) of the pancreatic tissue map compared with the pixels of parenchyma. Quantification of the nerve density is illustrated in Juang et al [13]. Briefly, feature extraction and image segmentation for quantitative analysis of the sympathetic nerve density were performed by the Label Field function of Avizo to collect the voxels of the pancreas and the associated $\mathrm{TH}^{+}$sympathetic nerves, which include the $\mathrm{TH}^{+}$axons and varicosities but exclude the globular $\mathrm{TH}^{+}$islet cells. Voxels of the nerves were divided by those of the pancreas $\times 100 \%$ to estimate the sympathetic nerve density. The same immunolabelling, imaging and quantification processes were conducted on the comparable pancreatic middle sections to characterise the tissue densities on the same basis.

Statistical analysis The quantitative values in Figs $2 \mathrm{f}, 4 \mathrm{a}$ and $6 \mathrm{e}-\mathrm{h}$ are presented as means and distribution of data points. Statistical differences were determined by the unpaired
Student's $t$ test. Differences between groups were considered statistically significant when $p<0.05$.

\section{Results}

\section{D panoramic histology of mouse pancreas}

We targeted the pancreas of 3-week-old weaning B6 mice to develop 3D panoramic histology. The decision to use these mice was based on two considerations. First, at this stage the mouse islets reach the end of their postnatal development and represent the functional endocrine tissues in the pancreas. Second, the pancreas is of a reasonable size (Fig. 1a, approximately $12 \times 9 \times 3 \mathrm{~mm}$ in an agarose block) to allow for tilescanning confocal microscopy to constitute the tissue map of the pancreas.

To enable efficient tissue clearing and microscopy, we systematically prepared a set of $500 \mu \mathrm{m}$ vibratome sections of the whole pancreas in specimen preparation. Examples of the pancreatic section before and after tissue clearing are presented in Fig. 1b, c. The depth of each section is 100-times thicker than the standard microtome section, allowing the spatial information of the nerves and islets to be preserved in the tissue block (electronic supplementary material [ESM] Video 1). However, we expected discontinuity of tissue information at the boundaries of the adjacent sections, which is a trade-off for effective imaging.

The transparent pancreas allows us to combine transmitted light and fluorescence imaging to construct the tissue map shown in ESM Fig. 1 (enlarged in ESM Video 1) and Fig. $1 d$ via tile scanning. Through image projection, Fig. 1d presents the TUJ1 (neuron-specific class III $\beta$-tubulin)-labelled nerve trunk which enters the pancreas and associates with the artery in extension. In addition, prominent nerve tracks are identified in the head and tail of the pancreas, which constitute the intra-pancreatic neural network.

Islet distribution, neurovascular association and population analysis in weaning mice Islets are scattered in the pancreas; however, they are not randomly scattered in space in weaning mice. Figure $2 \mathrm{a}$, b shows the typical peri-arteriolar neuro-insular aggregation in the pancreatic parenchyma, featuring the islets (particularly those $>50 \mu \mathrm{m}$ ) and TUJ1labelled nerve tracks spreading and extending longitudinally along the arteriole. The peri-arteriolar islet and nerve distribution can be found in all the pancreatic sections and is confirmed with the paired insulin and PGP9.5 (a second neuronal marker [20]) staining that provides an independent assessment (ESM Fig. 2). In addition, staining of the pancreas with the parasympathetic marker vAChT and sympathetic marker $\mathrm{TH}$ confirms that the autonomic nerves follow the arterioles and capillaries to reach the islet mantle and core (Fig. 2c-e). 

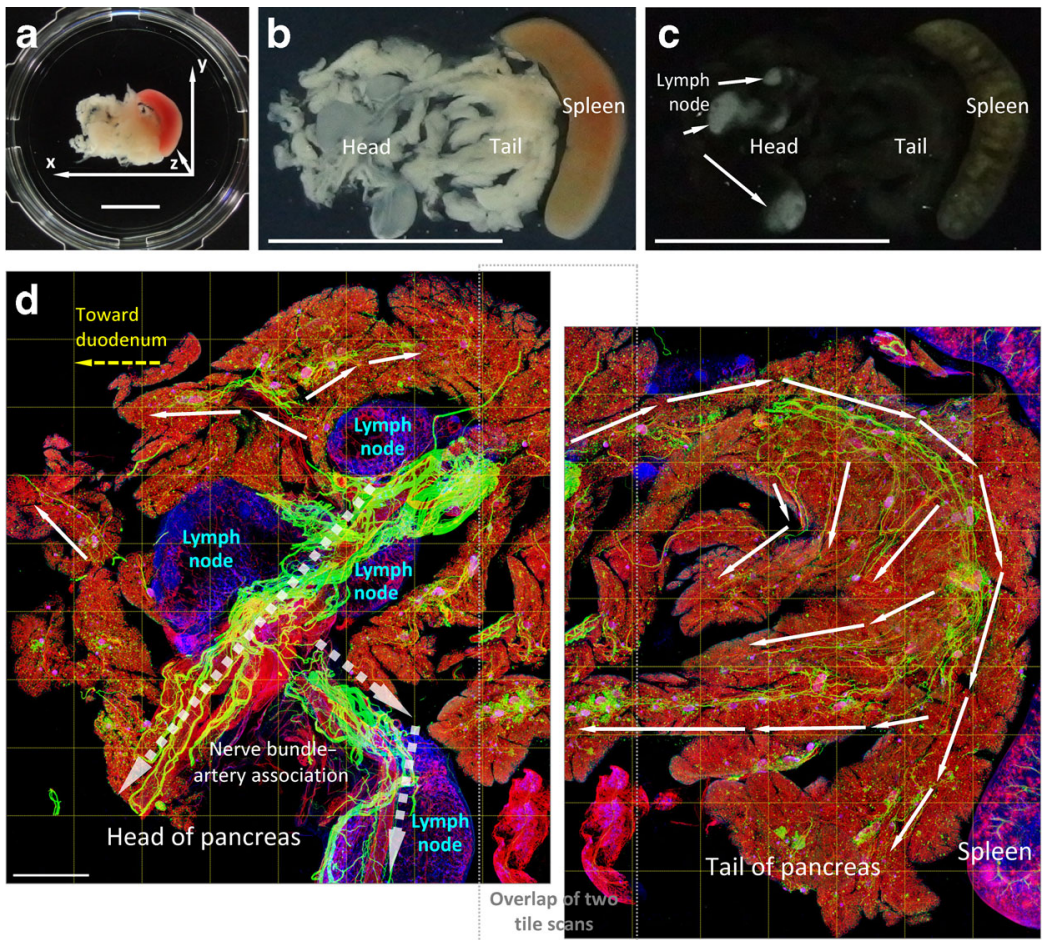

Fig. 1 Pancreatic tissue preparation and 3D panoramic histology. Pancreatic tissue clearing of a pancreas from a 3-week-old mouse. (a) Dissected whole pancreas. (b) Opaque pancreatic section. (c) Optically cleared section. Pancreatic section in (b) was acquired via vibratome sectioning along the $z$-axis direction shown in (a). The transparent specimen in (c) was derived from (b) after tissue clearing. Scale bars, $1 \mathrm{~cm}$. (d) Pancreatic tissue map derived from tile-scanned images of (c). Green,

Overall, these results indicate: (1) the dependence of islets on the feeding arteriole, (2) the presence of a neuro-insular network and (3) that islets are primary innervation targets in the postnatal development of the pancreas.

In whole pancreas islet quantification, the average numbers of the large (major axis $>150 \mu \mathrm{m}$ ), medium $(50-150 \mu \mathrm{m})$ and small (from a single cell at $10 \mu \mathrm{m}$ to $50 \mu \mathrm{m}$ ) islets identified in the atlases of the pancreases from two 3-week-old mice were 127, 633 and 7625, respectively. Using the middle pancreatic section as the area of interest (e.g. Fig. 1b, c), we analysed the density and tissue volume of the large, medium and small islets (Fig. 2f, g; $n=7$ mice). Significantly, while the density (or number) of the large islets is $<3 \%$ of all islets in the pancreas, they contribute $>70 \%$ of the overall islet volume in the weaning mice (Fig. $2 \mathrm{~g}$ ).

\section{Pancreatic innervation and ganglionic population in} weaning mice The pancreas receives autonomic innervation to regulate endocrine and exocrine functions. Figure 3 a shows the gross view of the pancreatic nerve import and its relay into the parenchyma. When focused on the network, Fig. 3b shows the relay and the nerve tracks that couple the islets and ganglia to establish the islet-ganglionic association. (Note that in Fig. neuronal TUJ1 staining; red, vascular staining; blue, insulin staining. Islets appear in magenta (overlap of red and blue) in the pancreatic parenchyma. Both spleen and lymph nodes show insulin staining background. Dashed arrows indicate the nerve trunk-artery association and their elongation in the head of pancreas. Solid arrows highlight the nerve tracks of the intra-pancreatic neural network. Scale bar, $1 \mathrm{~mm}$

$3 \mathrm{~b}$ numbers have been added to identify the ganglia, highlighting their presence and aggregation with the islets.)

In whole pancreas ganglionic quantification, 258 and 210 ganglia were identified in the atlases of the pancreases from two 3-week-old mice. Importantly, through TUJ1 staining and panoramic imaging, large ganglia $(>100 \mu \mathrm{m})$ and islets are

Fig. 2 Islet distribution, neurovascular association and population analysis in weaning mice. (a) Peri-arteriolar neuro-insular aggregation. Inset: photo of pancreatic section. White box indicates the area enlarged in (b). Green, neuronal TUJ1 staining; red, vascular staining; blue, insulin staining; magenta, islets. Scale bar, $1 \mathrm{~mm}$. (b) Magnified examination of peri-arteriolar islet distribution. Islets are found spreading along the longitudinal direction of the feeding arteriole (arrows). Scale bar, $1 \mathrm{~mm}$. (c-e) Peri-arteriolar autonomic nerves and their association with islets. (c, d) Parasympathetic marker vAChT staining (varicosities appear as white dots along the axon). Scale bars, $200 \mu \mathrm{m}$. (e) Sympathetic marker TH staining (axons and varicosities in yellow). Scale bar, $500 \mu \mathrm{m}$. Note that vAChT staining also reveals the peri-islet ganglia (arrows); TH staining shows the $\mathrm{TH}^{+}$islet cells. (f, g) Density, size distribution and relative tissue volume of large, medium and small islets. Size of islet is determined by its major axis shown in projection. In volume adjustment, we assume that the relative volumes of the large, medium and small islets are at the scale of 1000:125:1. The overall islet volume of a size group $=$ number of islets $\times$ relative volume. Data are derived from the islets in the middle pancreatic section (such as in Fig. 1c) of seven mice. Horizontal lines in (f) indicate the average of data points. Red, large islets; green, medium islets; blue, small islets 

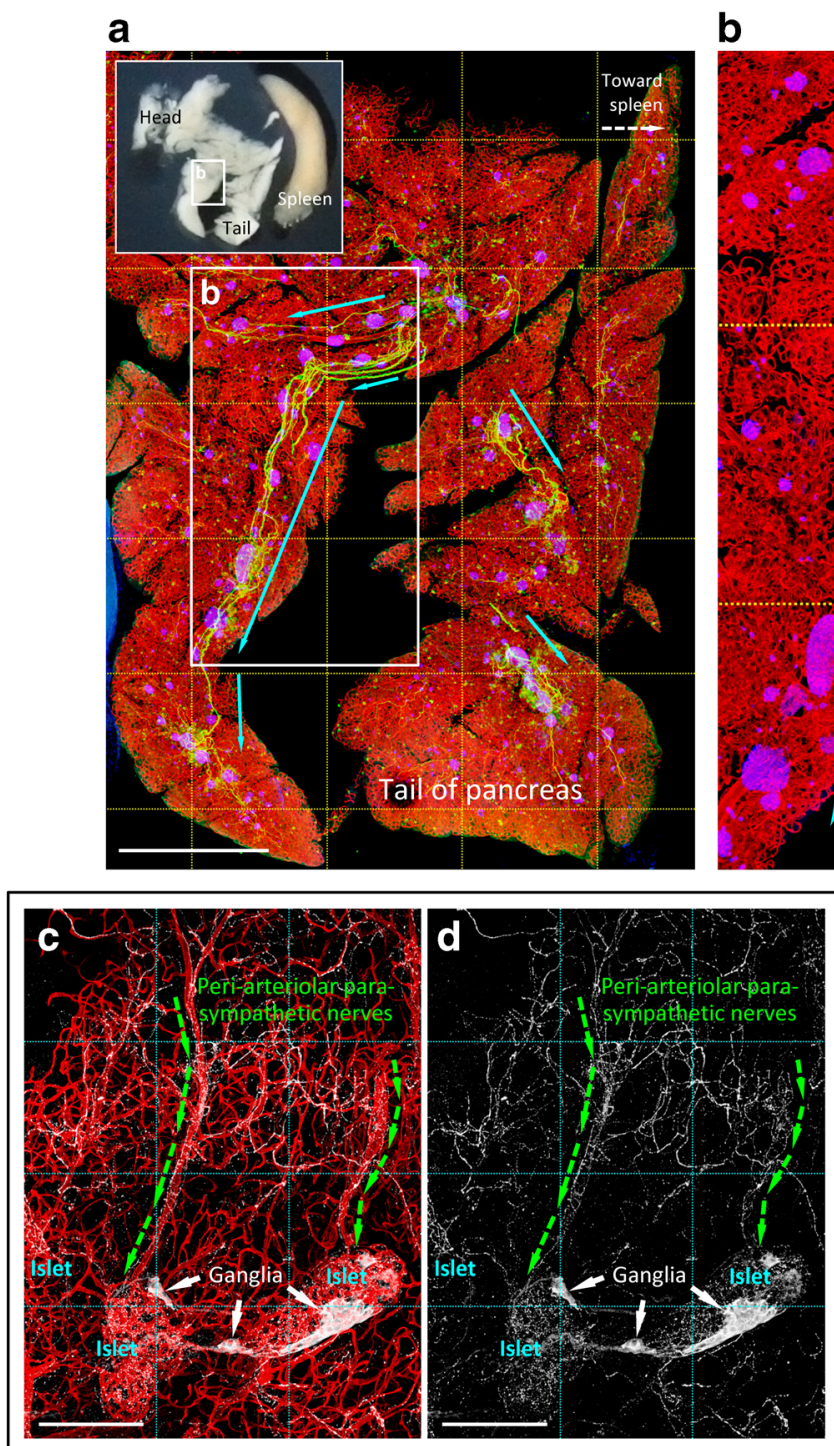

f

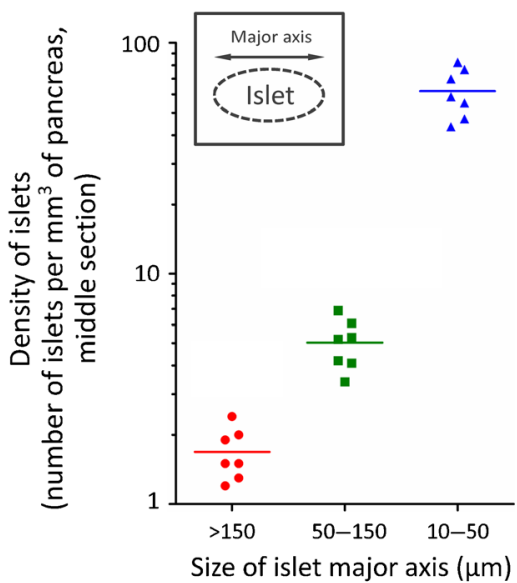

b
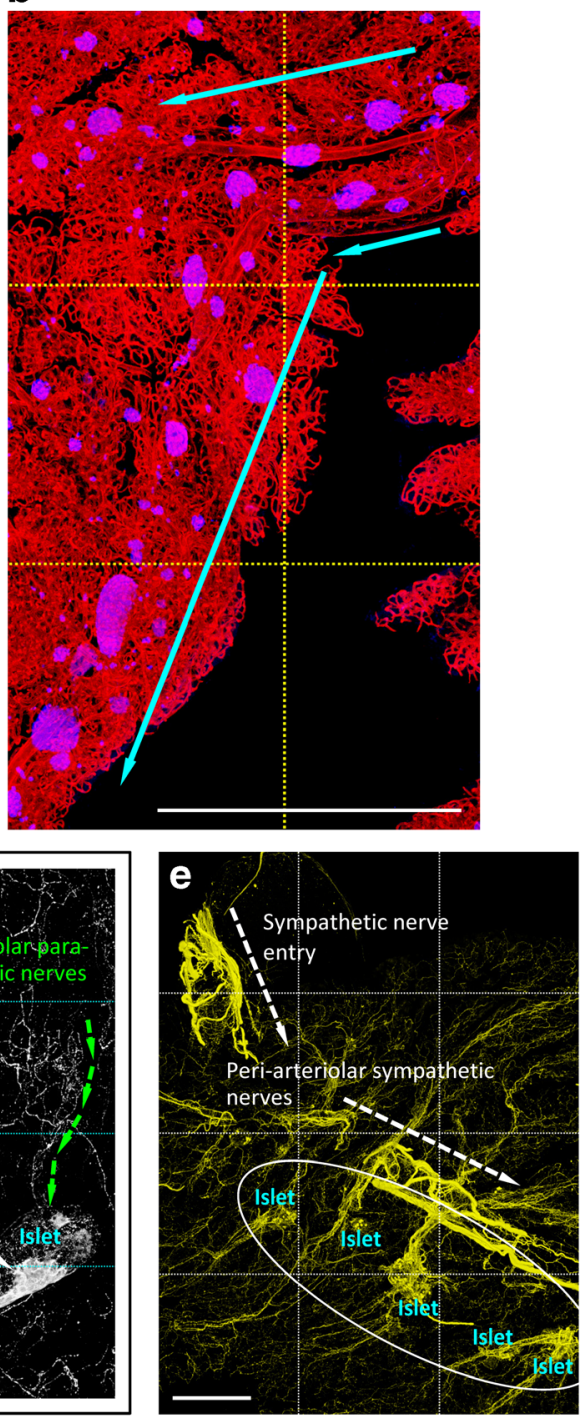

g

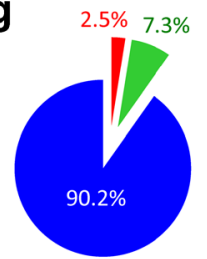

Size

distribution

Volume
adjustment

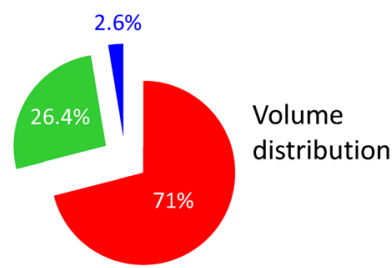


a

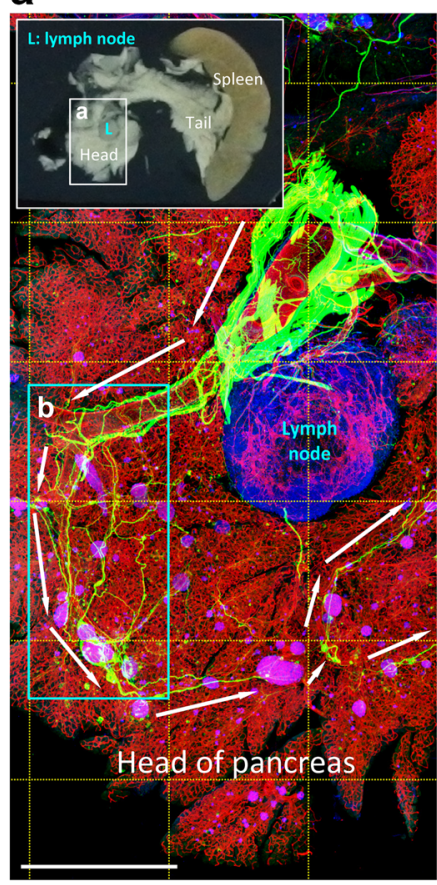

b

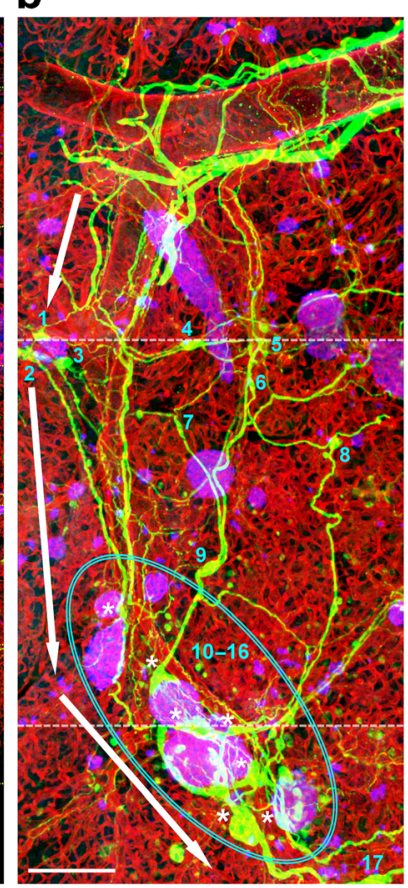

Fig. 3 Pancreatic nerve import and islet-ganglionic association in weaning mice. (a) Gross view of pancreatic nerve import and its relay into parenchyma. Green, neuronal TUJ1 staining; red, vascular staining; blue, insulin staining; magenta, islets. Arrows denote the entry and extensions of the TUJ1-labelled nerve trunk and tracks. The cyan box indicates the area enlarged in (b). Scale bar, $1 \mathrm{~mm}$. (b) Islet-ganglionic association and relay of the pancreatic neural network. Islets and ganglia are coupled with the TUJ1-labelled nerve tracks to form the network. A ganglion is defined as the $\mathrm{TUJ} 1^{+}$swelling body on the neural network with at least two nerve projections from the body. Seventeen ganglia are identified in this image and labelled with numbers. The oval indicates a cluster of islets and ganglia (numbers 10-16). Asterisks denote the ganglia identified in this region. Scale bar, $200 \mu \mathrm{m}$

readily recognised as landmarks of the neuro-insular network. Using the middle pancreatic section as the area of interest, we estimate that the large ganglia account for $12 \%$ of the ganglionic population (Fig. $4 \mathrm{a} ; n=6$ mice).

In addition to the size difference, the intra-pancreatic ganglia also vary in the number of nerve projections. The majority of the ganglia ( $81 \%$ ) have two to four projections (Fig. 4b), while the remaining $19 \%$ have five to eight projections, indicating the heterogeneity of the ganglionic population in the pancreatic neural network. Specifically, within the population of the large ganglia (Fig. 4c), we identified the presence of hub ganglia (71\%), which possess a body $>100 \mu \mathrm{m}$ and five to eight radiant projections in space. Figure 4d and ESM Video 2 present the hub ganglia and a detailed view of the ganglionic body and projections.

Islet-ganglionic association preserved in the process of maturing Next we compared the pancreases of 3- and 8week-old mice to investigate the islet-ganglionic association during maturation (post-weaning development of body and

pancreatic size). Similar to the neuro-insular network in the 3-week-old mice, we also identified peri-arteriolar aggregation of islets and ganglia in 8-week-old mice and formation of a network (Fig. 5a, b). The neuro-insular network features the $\mathrm{TUJ} 1^{+}$neural plexus coupling the islets and ganglia (both are peri-lobular) to establish the islet-ganglionic association. A gallery display of the association is presented in ESM Fig. 3. The neuro-insular network and islet-ganglionic association indicate that in the post-weaning phase of pancreatic development endocrine islets are the target of neural regulation.

In the post-weaning phase of development, however, the pancreas continues to grow. Quantitative analysis of islet aggregation indicates that large islets in pancreases from 8 -week-old mice are not as intimate to each other as those in the 3-week-old mice (Fig. $5 c)$. This appears to be caused by exocrine expansion. In the meantime, we used two indicators to evaluate the islet-ganglionic association: (1) the percentage of large islets within $100 \mu \mathrm{m}$ of a ganglion (Fig. 5d); and (2) the percentage of large ganglia within $100 \mu \mathrm{m}$ of a large/medium islet (Fig. 5e) (note: $100 \mu \mathrm{m}$ was used as an arbitrary cut-off distance for quantitative assessment). In both tests, we found that the exocrine expansion only marginally affected the association, indicating an intrinsic affinity of neural tissues (nerves and ganglia) with islets in the pancreas.

Adipose-pancreatic complex and sympathetic nerve remodelling in weaning $\boldsymbol{d} \boldsymbol{b} / \boldsymbol{d} \boldsymbol{b}$ mice The $d b / d b$ mice develop hyperphagic obesity due to a mutation in the satiety hormone leptin receptor in the hypothalamus [21]. At 3 weeks of age, the weaning $d b / d b$ mice already have recognisable weight gain and excess fat deposits in the abdomen, including the adipose tissue associated with the pancreas. Although hyperphagia contributes to the obesity, the lack of functional leptin signals to stimulate lipolysis is also linked to adipose tissue development [22, 23].

It has been established that leptin signalling deficiency reduces sympathetic tone, thereby diminishing lipolysis of white adipose tissue [24, 25]. However, the response of local sympathetic innervation in the pancreas to increases in surrounding adipose tissue and decreased sympathetic tone remains unclear.

To study the pancreas from $d b / d b$ mice, we applied 3D panoramic histology to examine the adipose-pancreatic complex in the weaning mice. Figure $6 \mathrm{a}$, b shows that the sympathetic nerves follow the vascular supply into the tissue complex and continue with the perivascular elongation to innervate the islets along the feeding arterioles. Importantly, compared with weaning $d b /+$ mice (lean control), the hyperphagic $d b / d b$ mice of the same age have markedly increased weight, adipose tissue attached to the pancreas, density of large islets (major axis $>150 \mu \mathrm{m}$ ) and pancreatic sympathetic nerve density (Fig. $6 \mathrm{c}-\mathrm{h}$ ). The results indicate that although sympathetic tone is downregulated in the $d b / d b$ mice, the local pancreatic sympathetic innervation is increased in association with adipose tissues and large islets in the hyperphagic weaning mice. 
Fig. 4 Intra-pancreatic ganglia in weaning mice: density, size and projections. (a) Density and relative amount of large and small ganglia in the pancreas. Line indicates the average density of ganglia identified in the middle sections of six mice. Red, large ganglia; blue, small ganglia. Size of a ganglion is determined by its major axis shown in projection. (b-d) Classification of ganglia based upon number of nerve projections and size. (b, c) 569 ganglia (including 66 large ganglia with major axis $>100 \mu \mathrm{m}$ ) in the middle sections from six mice were used in analysis. (d) Numbers 1-3 are three examples of a hub ganglion. Inset at the upper-left corner outlines the pancreatic section. Green, neuronal TUJ1 staining; red, vascular staining; blue, insulin staining; magenta, islets. Scale bars: main image, $500 \mu \mathrm{m}$; top inset, $2 \mathrm{~mm}$; bottom inset $100 \mu \mathrm{m}$ a

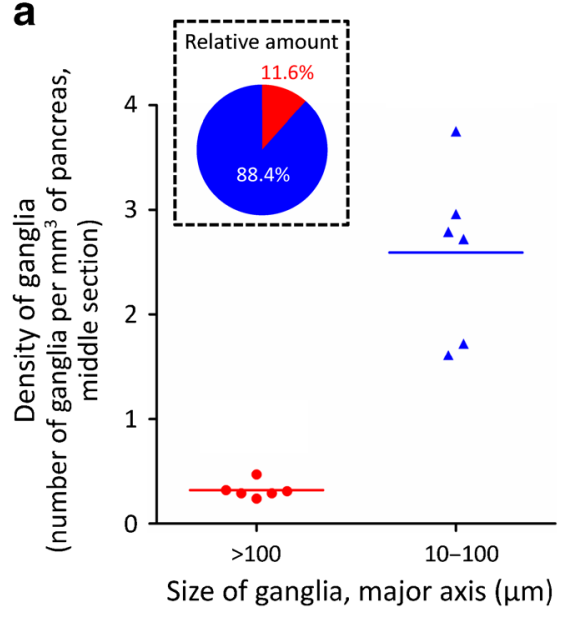

b

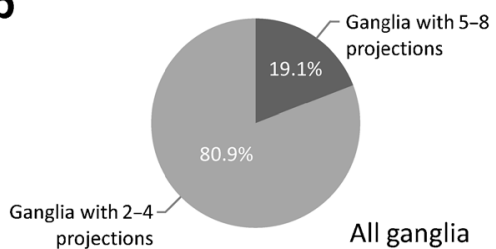

C

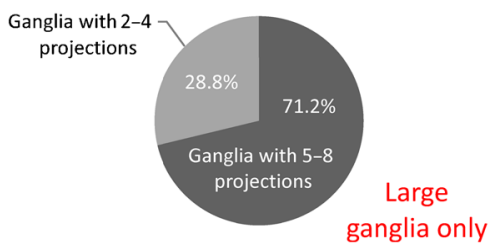

d

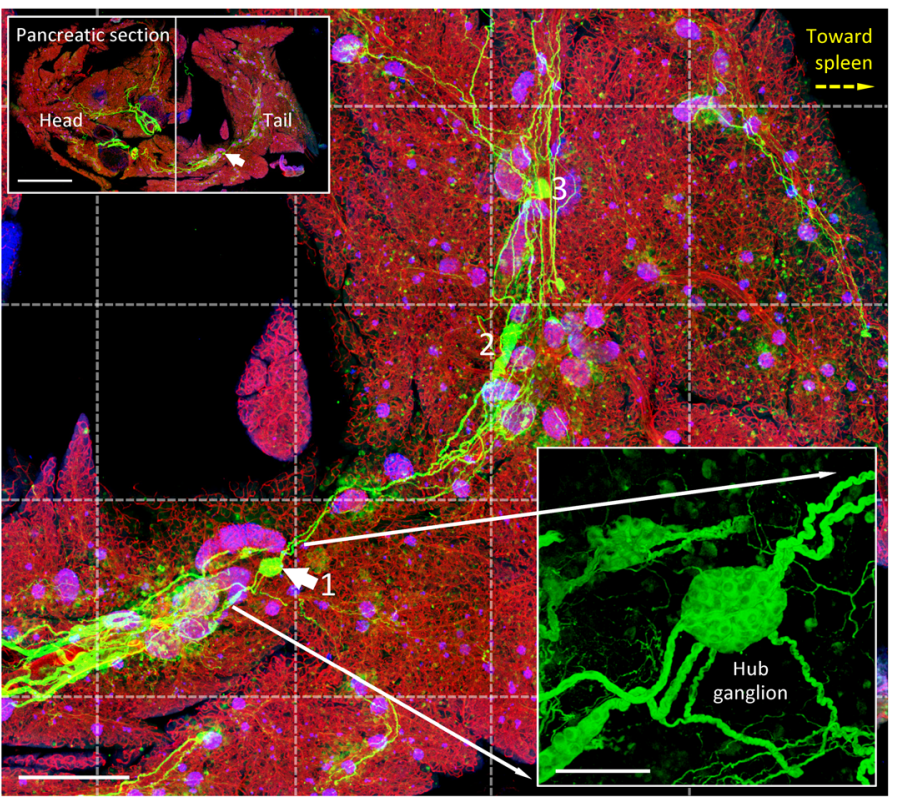

\section{Discussion}

We developed 3D panoramic histology to examine the pancreatic microstructure, vasculature and innervation in an integrated fashion in mice. The 3D imaging approach revealed the neuro-insular network at the organ level, which otherwise could not be visualised globally via microtome-based histology. In network characterisation, tile scanning and image stitching were used to generate a pancreatic tissue map, which depicts islet distribution and the pancreatic neurovascular network in a $3 \mathrm{D}$ space continuum. In disease characterisation, this analytical method identified the associated increases in weight, pancreatic adipose tissue, density of large islets and pancreatic sympathetic innervation in weaning hyperphagic $d b / d b$ mice.

The pancreatic neuro-insular network revealed in Figs 2, 3, 4,5 shows an intrinsic and intimate islet-ganglionic association during postnatal development and the process of maturation. Both the pancreatic nerve import (Fig. 3a; including the sympathetic nerves in Figs 2e, 6b) and the intra-pancreatic neural network (Figs 1d, 2a, 4d, 5a) are identified in this research, suggesting potential routes of exterior (brain-to-pancreas) and interior (pancreatic ganglion-to-islet) neural inputs to islets for modulation of hormone secretion [26-29]. The morphological evidence of the neuro-insular network supports the concept that despite being scattered in the pancreas the islets are coupled with the intra-pancreatic ganglia to coordinate their insulin secretion activity. In addition, the discovery of hub ganglia (Fig. 4d) further suggests that the intra-pancreatic neural network could possess hot spots that engage in local neural activity, rather than homogeneously innervating the pancreas for signal relay.

The imaging approach applied in this research is designed to be scalable for examination of mouse pancreases ranging from 
a

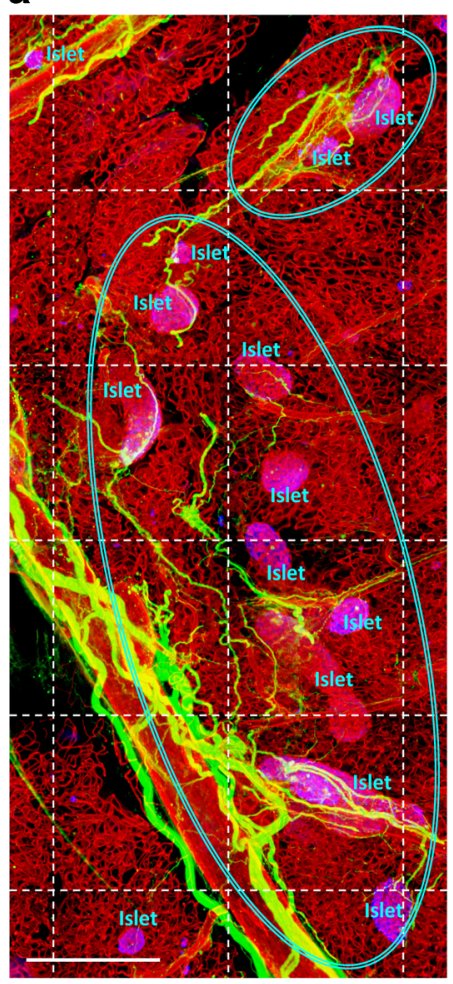

b

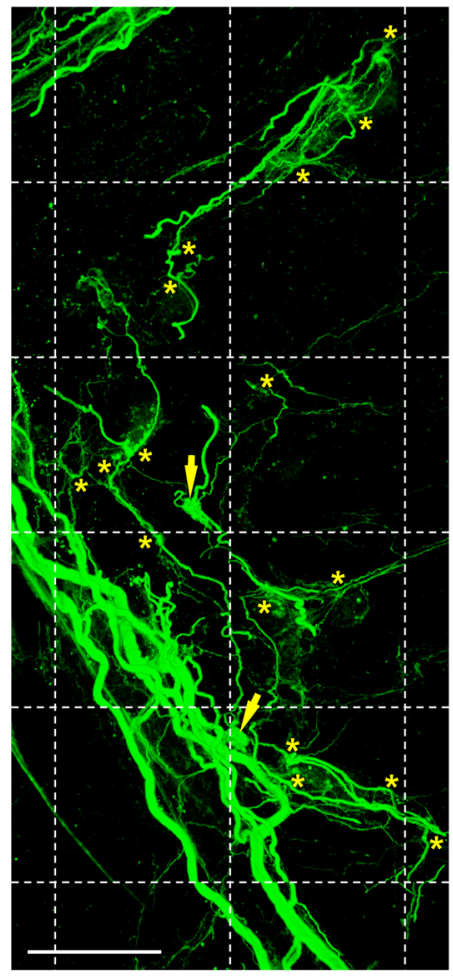

C Large islets away from other large/medium islets

n Large islets within $100 \mu \mathrm{m}$ of another large/medium islet
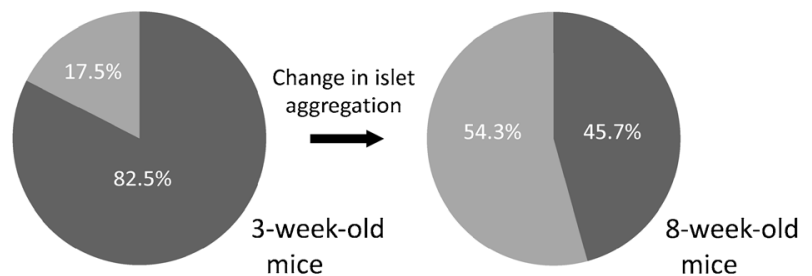

d Large islets away from ganglia

- Large islets within $100 \mu \mathrm{m}$ of a ganglion
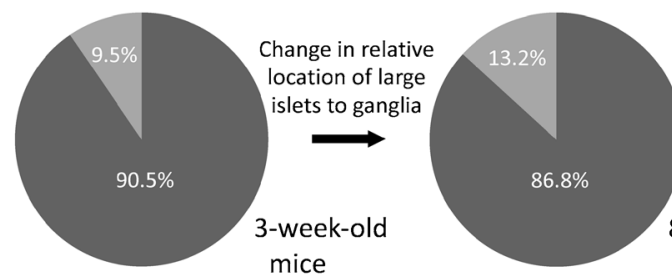

8-week-old mice

Large ganglia away from large/medium islets

Large ganglia within $100 \mu \mathrm{m}$ of a large/medium islet
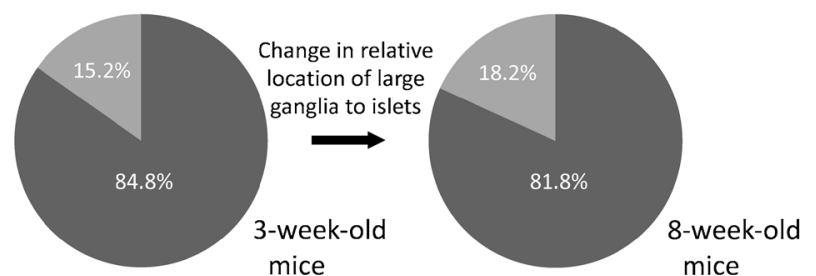

mice
Fig. 5 Islet-ganglionic association preserved in the process of maturing, 3-week-old vs 8-week-old mice. (a, b) Pancreatic peri-arteriolar neuroinsular network in 8-week-old mice. The oval in (a) represents peri-arteriolar islet and ganglionic aggregation. Asterisks in (b) represent ganglia. The arrows represent hub ganglia. Additional examples of the islet-ganglionic association are presented in ESM Fig. 3. Scale bars, $500 \mu \mathrm{m}$. (c) Quantitative analysis of islet aggregation, 3-week-old vs 8-week-old mice. Fewer large islets (45.7\%) are within $100 \mu \mathrm{m}$ of another large/ medium islet in the 8 -week-old compared with 3 -week-old mice

newborns to adults and in health and disease. In the disease model, we identified an increase in pancreatic sympathetic innervation in weaning $d b / d b$ mice. This increase can be attributed to neurotrophic factors secreted from the associated adipose tissue and islets to recruit the sympathetic nerves [30-32]. The local increase in sympathetic innervation could cause sympathetic overactivity in the basal state, despite the reduced overall responsiveness to sympathetic stimuli in obesity [33].

In neurohistology, the technical advance of tissue clearing has enabled the preparation of transparent specimens for 3D visualisation of the neural network with high definition [34-36]. However, unlike the brain, the pancreas is often associated with the adipose tissue, such as in the adipose-pancreatic complex revealed in Fig. 6a. Because adipose tissues are hydrophobic in nature, they are susceptible to the clearing methods that involve the membrane removal treatment of amphipathic molecules, such as SDS treatment in the CLARITY method [37]. Thus, while applying the tissue-clearing reagent
$(82.5 \%)$. In the analysis, $100 \mu \mathrm{m}$ was used as an arbitrary cut-off distance to assess islet-islet aggregation. (d, e) Evaluation of islet-ganglionic association, 3-week-old vs 8-week-old mice. The percentage of large islets within $100 \mu \mathrm{m}$ of a ganglion (d) and the percentage of large ganglia within $100 \mu \mathrm{m}$ of a large/medium islet (e) are marginally affected (3.7\% and $3.0 \%$ decrease, respectively) in the process of maturation. In (c-e), 189 large islets and 46 large ganglia in seven 3-week-old mice, and 105 large islets and 33 large ganglia in five 8-week-old mice were used in the analysis. Sizes of islets and ganglia are defined in Figs 2f, $4 \mathrm{a}$

(or method), it is crucial to monitor changes in the pancreatic structure and to compare/correlate the fluorescence signals with the transmitted light and gross tissue images, as presented in this research, to avoid misinterpretation of the pancreatic microenvironment.

In summary, we prepared transparent pancreatic specimens for 3D histology to achieve panoramic visualisation of islets and the associated neurovascular network in space. Before this study, islets and pancreatic nerves were only examined locally without a clear picture of the islet-ganglionic association and the peri-arteriolar neuro-insular network. Here, our 3D imaging approach overcomes the technical limitations imposed by standard histology to characterise the pancreatic neural network in health and disease. The pancreatic tissue maps offer the blueprint for future research into the interplay among the pancreatic microstructure, vasculature and innervation to increase our understanding of the mechanisms that influence islet and pancreatic activities. 

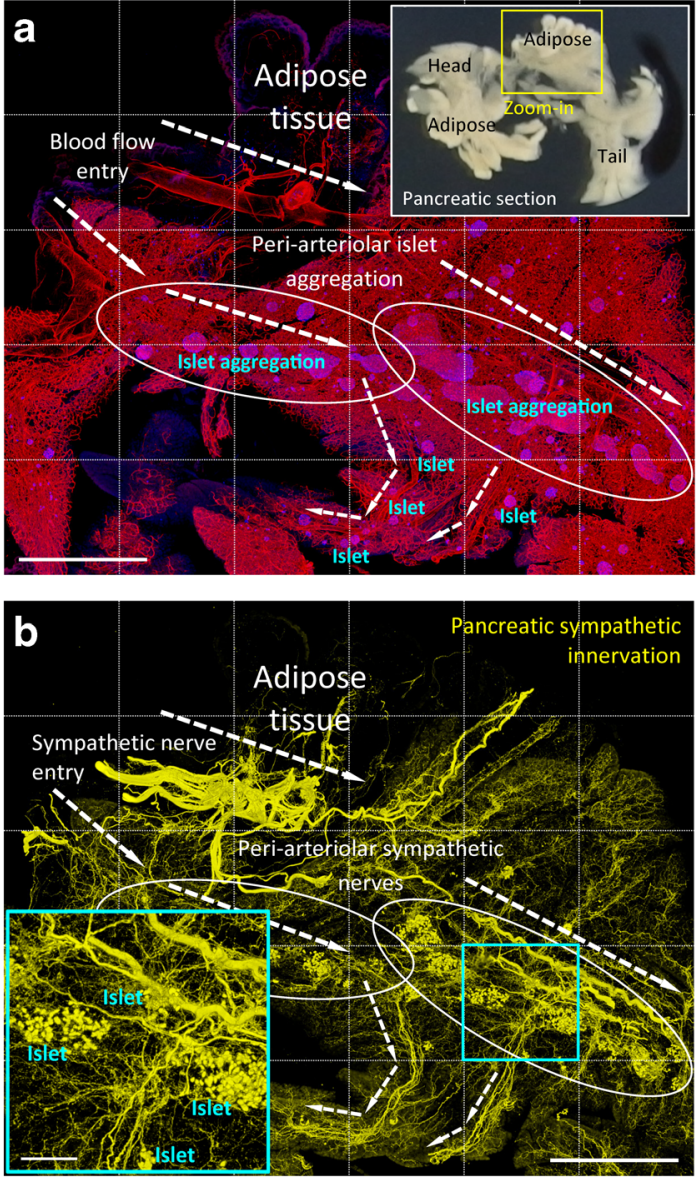

Fig. 6 Adipose-pancreatic complex and increase in pancreatic sympathetic innervation in weaning $d b / d b$ mice. $(\mathbf{a}, \mathbf{b})$ Adipose-pancreatic association, islet aggregation and sympathetic innervation in weaning $d b / d b$ mice. Inset in (a): pancreatic section showing the area presented in (a) and (b) (yellow box). (a) and (b) were taken under the same view. Dashed arrows indicate the directions of arterioles. Sympathetic nerves (b) follow the vascular system (a) into the adipose-pancreatic complex and elongate along the microvessels into the pancreatic parenchyma. The ovals indicate the areas with aggregated islets (a) and condensed sympathetic nerves (b). The nerves are enlarged in the inset (cyan box in $\mathbf{b}$; scale bar, $200 \mu \mathrm{m}$ ). (a, b) Main images, scale bars, $1 \mathrm{~mm}$. (c, d) Pancreatic

Acknowledgements We thank the confocal imaging core in National Tsing Hua University for technical support.

Data availability The images and datasets generated and/or analysed during the current study are available from the corresponding author on reasonable request.

Funding This work was supported in part by grants from the Taiwan Ministry of Science and Technology (MOST; 102-2314-B-001-006-MY3 and 105-2314-B-001-004) and Academia Sinica (intramural) to CNS; and the Taiwan National Health Research Institutes (NHRI-EX10410332EI and NHRI-EX105-10524EI) and MOST (104-2314-B-007002-MY2) to SCT.
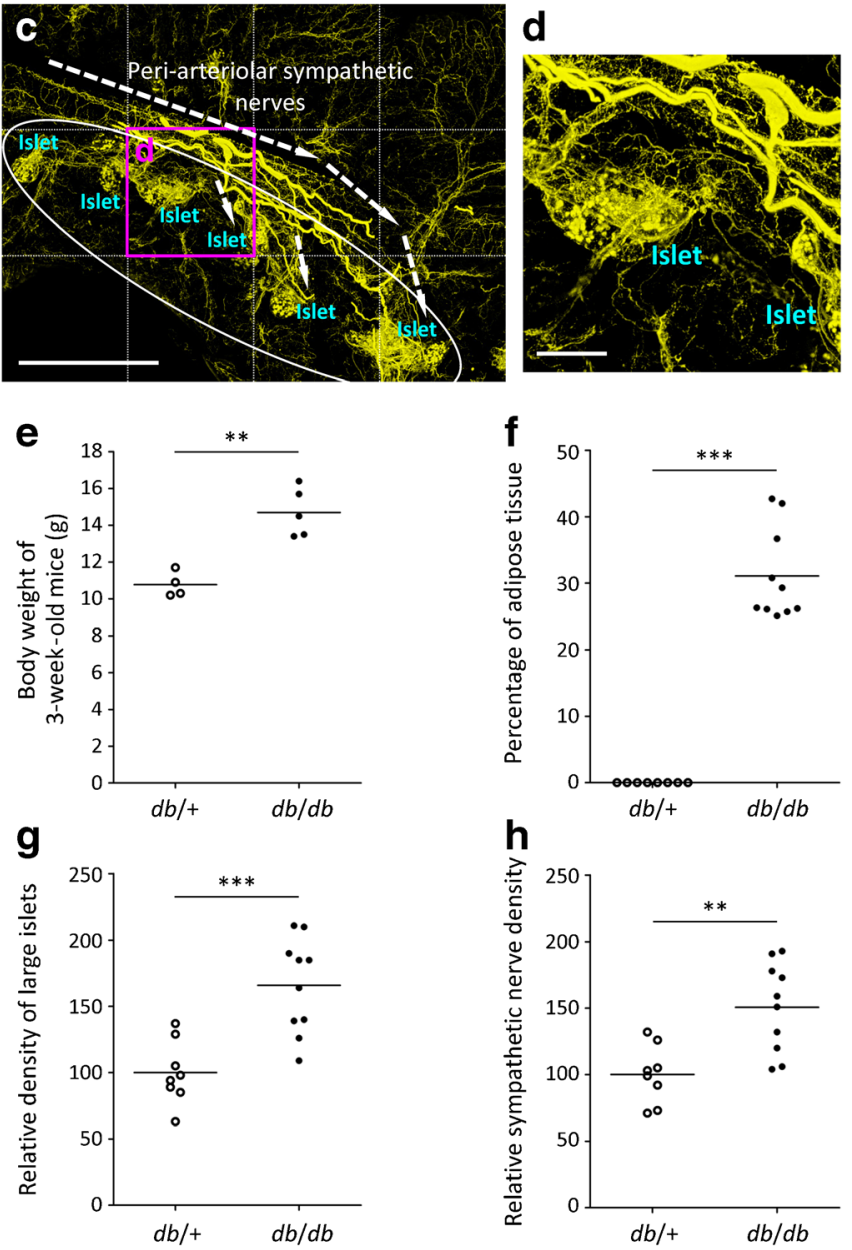

sympathetic innervation in weaning $d b /+$ mice (control). Peri-arteriolar islets (oval) and the sympathetic nerves are also prominent in the $d b /+$ pancreas but they are not as condensed as those in the $d b / d b$ pancreas. The magenta box in (c) is enlarged in (d). Scale bars: (c) $1 \mathrm{~mm}$; (d) $200 \mu \mathrm{m}$. (e-h) Associated increases in weight (e), adipose tissue associated with pancreas (f, expressed as [adipose/(adipose + parenchyma) $\times 100]$ ), large islet density (g, expressed as \% of $d b /+$ pancreas) and sympathetic nerve density (h, expressed as \% of $d b /+$ pancreas) in weaning $d b / d b$ mice. Data are derived from four $d b /+$ and five $d b / d b$ mice. In $(\mathbf{f}-\mathbf{h})$, two middle pancreatic sections from each mouse were used in the analysis. $* * p<0.01, * * * p<0.001$

Duality of interest The authors declare that there is no duality of interest associated with this manuscript.

Contribution statement All authors contributed to the study concept and design. CNS, PYL and SJP contributed to specimen acquisition. SCT, SJP, HJC and YHC contributed to 3D histology and image presentation. SCT, CNS, CEC and PJP contributed to drafting of the manuscript. All authors contributed to data analysis and interpretation of data, and revised the manuscript critically for intellectual content. All authors approved the final version of the manuscript. SCT and CNS obtained funding. SCT is the guarantor of this work and, as such, had full access to all the data in the study and takes responsibility for the integrity of the data and the accuracy of the data analysis. 


\section{References}

1. Langerhans $P$ (1869) Contributions to the microscopic anatomy of the pancreas. MD thesis, Berlin (translated by Morrison, H., 1937). John Hopkins Press, Baltimore

2. Ahren B (2000) Autonomic regulation of islet hormone secretionimplications for health and disease. Diabetologia 43:393-410

3. Ahren B, Holst JJ (2001) The cephalic insulin response to meal ingestion in humans is dependent on both cholinergic and noncholinergic mechanisms and is important for postprandial glycemia. Diabetes 50:1030-1038

4. Pirola L, Johnston AM, Van Obberghen E (2004) Modulation of insulin action. Diabetologia 47:170-184

5. Lang DA, Matthews DR, Peto J, Turner RC (1979) Cyclic oscillations of basal plasma glucose and insulin concentrations in human beings. N Engl J Med 301:1023-1027

6. Porksen N, Munn S, Steers J, Vore S, Veldhuis J, Butler P (1995) Pulsatile insulin secretion accounts for $70 \%$ of total insulin secretion during fasting. Am J Phys 269:E478-E488

7. Gylfe E, Tengholm A (2014) Neurotransmitter control of islet hormone pulsatility. Diabetes Obes Metab 16(Suppl 1):102-110

8. Porksen N (2002) The in vivo regulation of pulsatile insulin secretion. Diabetologia 45:3-20

9. Satin LS, Butler PC, Ha J, Sherman AS (2015) Pulsatile insulin secretion, impaired glucose tolerance and type 2 diabetes. Mol Asp Med 42:61-77

10. Lang DA, Matthews DR, Burnett M, Turner RC (1981) Brief, irregular oscillations of basal plasma insulin and glucose concentrations in diabetic man. Diabetes 30:435-439

11. Porksen N, Hollingdal M, Juhl C, Butler P, Veldhuis JD, Schmitz O (2002) Pulsatile insulin secretion: detection, regulation, and role in diabetes. Diabetes 51:S245-S254

12. Borden P, Houtz J, Leach SD, Kuruvilla R (2013) Sympathetic innervation during development is necessary for pancreatic islet architecture and functional maturation. Cell Rep 4:287-301

13. Juang JH, Peng SJ, Kuo CH, Tang SC (2014) Three-dimensional islet graft histology: panoramic imaging of neural plasticity in sympathetic reinnervation of transplanted islets under the kidney capsule. Am J Physiol Endocrinol Metab 306:E559-E570

14. Juang JH, Kuo CH, Peng SJ, Tang SC (2015) 3-D imaging reveals participation of donor islet Schwann cells and pericytes in islet transplantation and graft neurovascular regeneration. EBioMedicine 2:109-119

15. Chiu YC, Hua TE, Fu YY, Pasricha PJ, Tang SC (2012) 3-D imaging and illustration of the perfusive mouse islet sympathetic innervation and its remodelling in injury. Diabetologia 55:3252-3261

16. Tang SC, Chiu YC, Hsu CT, Peng SJ, Fu YY (2013) Plasticity of Schwann cells and pericytes in response to islet injury in mice. Diabetologia 56:2424-2434

17. Fu YY, Lu CH, Lin CW et al (2010) Three-dimensional optical method for integrated visualization of mouse islet microstructure and vascular network with subcellular-level resolution. J Biomed Opt 15:046018

18. Lin PY, Peng SJ, Shen CN, Pasricha PJ, Tang SC (2016) PanINassociated pericyte, glial, and islet remodeling in mice revealed by
3-D pancreatic duct lesion histology. Am J Physiol Gastrointest Liver Physiol 311:G412-G422

19. Chien HJ, Peng SJ, Hua TE, Kuo CH, Juang JH, Tang SC (2016) 3$\mathrm{D}$ imaging of islets in obesity: formation of the islet-duct complex and neurovascular remodeling in young hyperphagic mice. Int $\mathrm{J}$ Obes 40:685-697

20. Fu YY, Peng SJ, Lin HY, Pasricha PJ, Tang SC (2013) 3-D imaging and illustration of mouse intestinal neurovascular complex. Am J Physiol Gastrointest Liver Physiol 304:G1-G11

21. Chen H, Charlat O, Tartaglia LA et al (1996) Evidence that the diabetes gene encodes the leptin receptor: identification of a mutation in the leptin receptor gene in $d b / d b$ mice. Cell 84:491-495

22. Siegrist-Kaiser CA, Pauli V, Juge-Aubry CE et al (1997) Direct effects of leptin on brown and white adipose tissue. J Clin Invest 100:2858-2864

23. Zeng W, Pirzgalska RM, Pereira MM et al (2015) Sympathetic neuro-adipose connections mediate leptin-driven lipolysis. Cell 163:84-94

24. Lustig RH (2006) Childhood obesity: behavioral aberration or biochemical drive? Reinterpreting the First Law of Thermodynamics. Nat Clin Pract Endocrinol Metab 2:447-458

25. Nonogaki K (2000) New insights into sympathetic regulation of glucose and fat metabolism. Diabetologia 43:533-549

26. Thorens B (2014) Neural regulation of pancreatic islet cell mass and function. Diabetes Obes Metab 16(Suppl 1):87-95

27. Berthoud HR, Powley TL (1991) Morphology and distribution of efferent vagal innervation of rat pancreas as revealed with anterograde transport of Dil. Brain Res 553:336-341

28. Sha L, Westerlund J, Szurszewski JH, Bergsten P (2001) Amplitude modulation of pulsatile insulin secretion by intrapancreatic ganglion neurons. Diabetes 50:51-55

29. Fendler B, Zhang M, Satin L, Bertram R (2009) Synchronization of pancreatic islet oscillations by intrapancreatic ganglia: a modeling study. Biophys J 97:722-729

30. Sornelli F, Fiore M, Chaldakov GN, Aloe L (2009) Adipose tissuederived nerve growth factor and brain-derived neurotrophic factor: results from experimental stress and diabetes. Gen Physiol Biophys 28:179-183

31. Rosenbaum T, Vidaltamayo R, Sanchez-Soto MC, Zentella A, Hiriart M (1998) Pancreatic beta cells synthesize and secrete nerve growth factor. Proc Natl Acad Sci U S A 95:7784-7788

32. Vidaltamayo R, Mery CM, Angeles-Angeles A, Robles-Diaz G, Hiriart M (2003) Expression of nerve growth factor in human pancreatic beta cells. Growth Factors 21:103-107

33. Tentolouris N, Liatis S, Katsilambros N (2006) Sympathetic system activity in obesity and metabolic syndrome. Ann N Y Acad Sci 1083:129-152

34. Richardson DS, Lichtman JW (2015) Clarifying tissue clearing. Cell 162:246-257

35. Tang SC, Peng SJ, Chien HJ (2014) Imaging of the islet neural network. Diabetes Obes Metab 16(Suppl 1):77-86

36. Susaki EA, Tainaka K, Perrin D et al (2014) Whole-brain imaging with single-cell resolution using chemical cocktails and computational analysis. Cell 157:726-739

37. Chung K, Wallace J, Kim SY et al (2013) Structural and molecular interrogation of intact biological systems. Nature 497:332-337 